\title{
LABORATORIOS DE INNOVACIÓN PARA CAMBIAR LA GESTIÓN PÚBLICA: ANÁLISIS DEL CASO NOVAGOB.LAB
}

\author{
J. Ignacio Criado \\ Universidad Autónoma de Madrid, España \\ ignacio.criado@uam.es \\ Francisco Rojas-Martín \\ Novagob, España \\ frojas@novagob.org \\ Aitor Silván \\ Novagob, España \\ aitor@novagob.org
}

\section{RESUMEN}

Los laboratorios de innovación (innovation labs o living labs) son espacios diseñados para promover la transformación de la gestión pública. Este artículo se acerca a los laboratorios de innovación pública mediante un estudio de caso: NovaGob Lab, Laboratorio de Gobierno para la Innovación Púbica, el primero de este tipo creado en España. Así, a partir del análisis de una comunidad de innovación en el ámbito de la planificación estratégica para el sector público, se contrasta el potencial de este tipo de instrumentos para desarrollar innovaciones en la gestión pública, mediante la aplicación de metodologías experimentales y la utilización de dinámicas de co-creación y colaboración en las organizaciones, entre actores internos y externos. En definitiva, se extraen conclusiones de interés para entender las implicaciones de los laboratorios de innovación para cambiar las administraciones públicas, comprender las nuevas vías de co-creación con la ciudadanía en la solución de los problemas sociales, así como contextualizar el nuevo paradigma de gobernanza inteligente en la gestión pública.

Palabras clave: Innovación abierta, Gestión pública, Co-producción, Colaboración, Laboratorio, Design-thinking, 


\title{
INNOVATION LABORATORIES TO CHANGE PUBLIC MANAGEMENT: ANAYSIS OF THE NOVAGOB.LAB CASE
}

\begin{abstract}
Innovation labs or living labs are spaces designed to promote the transformation of public management. This paper approaches innovation laboratories through a case-study: NovaGob Lab, Government Laboratory for Pubic Innovation - the first of its kind in Spain. Thus, from the analysis of an innovation community in strategic planning for the public sector, the potential of these kinds of instruments for developing innovation in public management is contrasted, through the application of experimental methodologies and the use of dynamics of co-creation and collaboration within organizations, among internal and external players. Conclusions are reached for understanding the implications of innovation laboratories for changing public administration, understanding the new avenues of cocreation with citizens in problem-solving, as well as for contextualizing the new paradigm of intelligent governance in public management.
\end{abstract}

Keywords: Open innovation, Public management, Co-production, Collaboration, Laboratory, Design-thinking. 


\section{INTRODUCCIÓN}

La innovación pública se ha convertido en uno de los ámbitos de interés de los académicos a nivel internacional dentro de la gestión pública. La necesidad de transformar las organizaciones para crear valor público (esto es, generar valor en términos de inclusión, participación, eficiencia, escalabilidad, sostenibilidad, o igualdad) ha llevado a la creación de entidades u organizaciones específicas encargadas de este tipo de actividades innovadoras (de Vries, Bekkers, y Tummers 2016, Cejudo, Dussauge y Michel 2016). Este artículo se centra en el papel de los laboratorios de innovación pública en la transformación de la gestión pública (innovation labs o living labs) (Tonurist, Kattel y Lember 2017) y analiza de manera comparativa con otras experiencias existentes a nivel internacional, el caso de NovaGob.Lab, Laboratorio de Gobierno para la Innovación Pública en Iberoamérica, el primero de esta naturaleza creado en España. Con ello se pretende, por un lado, conocer cuáles son los fundamentos de actuación de los laboratorios de innovación pública y, por otro lado, poner de relieve su capacidad para generar innovaciones reales para solucionar problemas públicos y promover dinámicas orientadas a una gobernanza inteligente (Criado 2016b). El conocimiento en profundidad de un caso de estudio ofrece la evidencia necesaria para extraer las conclusiones que se dibujan a lo largo de este trabajo.

Los laboratorios de innovación se han constituido en su mayoría como espacios de experimentación en las organizaciones públicas. Desde un punto de vista académico, existen muy pocos trabajos que se hayan acercado a la realidad de los laboratorios de innovación como nuevos escenarios orientados a la transformación de la gestión pública desde una lógica de apertura, participación y colaboración. Se trata de entidades, en muchos casos autónomas de las instancias gubernamentales, basadas en la generación de innovación, que se dedican a apoyar a las organizaciones públicas en la búsqueda de soluciones a problemas sociales mediante metodologías abiertas, que suelen poner en el centro a las personas. Lo relevante es que estas nuevas organizaciones perciben la innovación en el sector público como su principal tarea, de manera que se crean específicamente para desarrollarla conscientemente bajo metodologías orientadas a la acción. Todos ellos, pese a su corta trayectoria, se han consolidado como referentes en las administraciones públicas de medio mundo.

El estudio de caso que se presenta en este artículo tiene su origen en la red social NovaGob. NovaGob es una red social profesional orientada a las administraciones públicas, que ya supera los diez mil profesionales del sector público iberoamericano, y ha impulsado el primer laboratorio 
de innovación pública nacido en España, denominado NovaGob.Lab. El objetivo del NovaGob.Lab es crear un ecosistema de intercambio de conocimiento en torno a la innovación pública ligado a la investigación básica, asistiendo a las administraciones públicas iberoamericanas en su tarea de innovación con una filosofía clara, esto es, servir de puente entre el mundo académico y el sector público, transfiriendo conocimiento a través de la lógica de laboratorio: investigación básica teorizada en la gestión pública y conectada con los problemas prácticos, al mismo tiempo, empleando conocimiento empírico adquirido sobre la intervención en problemas reales que afectan a las personas y con ello enriquecer la disciplina de la gestión pública.

La idea de los laboratorios de innovación pública surge de la realidad de que en las sociedades actuales no solo hay un modelo nuevo de producción de servicios públicos detrás, sino también, nuevas formas de innovar y de entender cómo gobernar lo público. Como han puesto de manifiesto Criado y Villodre (2016), si las innovaciones en las organizaciones públicas han venido hasta ahora promovidas por departamentos reducidos en el sector público, el desarrollo de un modelo de administración pública colaborativa, implica que dicha co-producción y co-creación se haga de forma abierta con la ciudadanía y que, por tanto, se amplíen las perspectivas de la organización hacia una manera de trabajar fundamentada en la innovación. El concepto de innovación abierta planteado por Chesbrough (2012), aterriza en el sector público de la mano de trabajos como el de Windrum (2007), Mergel (2015), Mergel y Desouza (2013), Clark et al. (2013), que demuestran que la innovación también se produce en el sector público de una manera cada vez consciente y abierta al entorno. Más recientemente, Gil-García, Zhang y Purón (2016), Noveck (2015), Criado (2016b) o Rodríguez-Bolívar y Meijer (2015) utilizan también el concepto smart en la acción de gobierno, como elemento distintivo de la nueva manera de involucrar a la ciudadanía en los asuntos públicos, así como de dibujar la gobernanza de sociedades cada vez más complejas.

El resto del artículo se desarrolla a continuación con el siguiente esquema. En la siguiente sección se presenta un marco de referencia sobre la innovación en las administraciones públicas, teniendo en cuenta nuevos conceptos emergentes (tales como inteligencia colectiva, co-creación, co-producción, etc.), identificando a continuación de forma comparada algunos de los casos de laboratorio de innovación pública referentes en el ámbito internacional. Después se ofrece una aproximación al caso de NovaGob.Lab, identificando su relación con el proyecto NovaGob como ecosistema de innovación pública. En la siguiente sección se muestran evidencias reales tomadas de una intervención de NovaGob.Lab aplicando 
nuevas metodologías para enfrentar una problemática pública, a partir de las que se genera una solución basada en la lógica del prototipado (designthinking). Se cierra el trabajo con unas conclusiones acerca del papel de los laboratorios de innovación pública como espacios de experimentación, que pueden tener calado para el futuro de la gestión pública, en la medida de que se trate de procesos metodológicamente solventes, sólidamente teorizados y con experimentos bien diseñados.

\section{INNOVACIÓN PÚBLICA, INTELIGENCIA COLECTIVA Y LABORATORIOS DE INNOVACIÓN}

La utilización del término innovación dentro del sector público no es algo nuevo, si bien durante los últimos ańos se ha producido un incremento de su interés. Esto se encuentra conectado con la existencia de una nueva generación de tecnologías sociales y grandes cantidades de datos que permiten la acumulación de inteligencia colectiva y el desarrollo de nuevos formatos, tales como los laboratorios de innovación. De hecho, hay quienes asumen que la innovación en el sector público se puede entender como un nuevo campo de conocimiento (de Vries, Bekkers, y Tummers 2016). Particularmente, la generación de inteligencia colectiva derivada de la disponibilidad de grandes datos, captados a través de tecnologías de carácter social y cada vez más inter-conectadas, permite que las dinámicas de innovación organizativa en el ámbito de lo público se orienten al entorno, de manera que se hacen cada vez más abiertas a la ciudadanía (Linders 2012). Así, los laboratorios de innovación concretan todo ello al generar espacios de híbridos, en los que las administraciones públicas se abren a la ciudadanía para mejorar los procesos de adopción de decisiones e imaginar nuevas maneras de solucionar los problemas de la comunidad.

\section{InNOVACiÓn EN EL SECTOR PÚBLICO. AlgunAS NOTAS DE PARTIDA}

Aunque las administraciones públicas han innovado desde siempre, ya sea de manera fortuita, ocasionalmente o de manera continuada, el estudio sistemático de la innovación en el sector público es bastante reciente. Los esfuerzos se han equilibrado progresivamente a favor del sector público en los últimos diez años, pero recientes estudios muestran que existen diez veces más estudios sobre la innovación en el sector privado, que en el sector público (Rivera León, Simmonds y Roman 2012). De hecho, las administraciones públicas siguen una tradición garantista y burocrática, lo que significa que cuentan con una lógica organizativa rígida y piramidal. Este modelo administrativo no es el idóneo para generar ideas innovadoras. Por ello, para que la innovación pública no sea un proceso casuístico, singular y aleatorio, es esencial crear espacios adecuados para su desarrollo. 
En su revisión de literatura reciente, de Vries, Bekkers, y Tummers (2016) muestran que la innovación en el sector público ha crecido de manera importante y se orienta hacia diferentes dimensiones (o tipos). En concreto, estos autores seńalan la existencia de cuatro tipos de innovaciones en el sector público: 1) innovación en los procesos, bien sean de carácter administrativo, bien de carácter tecnológico; 2) innovación en los productos y servicios, sobre todo, mediante la creación de nuevos productos o servicios públicos; 3) innovación en la gobernanza, es decir, aquélla centrada en el desarrollo de nuevas formas de atender problemas sociales concretos; 4) innovación conceptual, que se centra en la introducción de nuevos conceptos, marcos de referencia o paradigmas que ayuden a reformular la naturaleza misma de los problemas, así como las posibles soluciones que se considera viable utilizar.

Entre las variantes planteadas no existe una homogeneidad en la aplicación dentro del sector público. De hecho, la mayoría de las innovaciones en las administraciones públicas se han centrado en la primera y segunda de las categorías, sobre todo, aquellas que siguen inercias de la Nueva Gestión Pública (NGP). Las innovaciones en la gobernanza y la innovación conceptual, que claramente tienen un carácter más sustantivo y superador de los planteamientos de la NGP (Brugué, Blanco y Boada 2014), no han contado con tanto interés (Vries, Bekkers, y Tummers 2016). Entre otras razones, lo anterior es debido a la propia novedad de un enfoque sobre la innovación que, en buena medida, supone la superación de dinámicas muy asentadas en la gestión de las organizaciones, en general, y las administraciones públicas, en particular.

INTELIGENCIA COLECTIVA, CO-CREACIÓN Y COLABORACIÓN EN LOS PROBLEMAS SOCIALES

La innovación en las administraciones públicas cada vez se encuentra más asociada a la colaboración con el entorno, la inteligencia colectiva y la co-creación. Linders (2012), Criado y Villodre (2016) o Sørensen yTorfing (2012) han planteado que la inteligencia colectiva o crowdsourcing, normalmente asociada a las nuevas tecnologías y plataformas sociales, es un proceso de co-creación de servicios que permite que, frente a la existencia de un problema, o la necesidad de colaboración en la construcción de una información pública (Brabham 2013): 1) la organización selecciona a un colectivo, más o menos formado (pero no a un club de expertos); 2) la comunidad se implica en la co-producción de la información o en la resolución conjunta de la problemática; 3) el resultado dispone de un beneficio dual y mutuo para organización y sabiduría colectiva. En otras palabras, se puede hablar de un proceso de problem-solving, un 
modelo de producción que consiste en una actividad participativa donde organizaciones, individuos, etc., proponen a una colectividad (más o menos experta e implicada en la problemática a tratar) la resolución voluntaria de un problema.

Por un lado, dentro de estas dinámicas, las nuevas tecnologías sociales permiten formas alternativas de creación de innovaciones sin la necesidad de tener tan presentes las reglas burocráticas y sus constreñimientos. De esa manera, según los beneficios que pueden resultar de la aplicación de tecnologías colaborativas en el sector público cuenta con el potencial para contribuir a mejorar, no solo la efectividad de los procesos administrativos y los servicios públicos, sino también para aumentar la transparencia de las dinámicas de innovación, así como reforzar los esfuerzos para acometer de forma colaborativa problemas sociales. Diferentes plataformas de participación ciudadana en línea (al estilo DecideMadrid o SantanderCityBrain en Espańa, así como Challenge.gov o GitHub en USA) estarían dentro de ese ámbito de acción (Criado 2016a; Mergel, 2015; Mergel y Desouza, 2013). Sin embargo, no todo son herramientas de colaboración en Internet, dado que ese tipo de procesos se puede activar de una forma más sofisticada dentro de espacios híbridos físicos diseñados para fomentar la innovación.

\section{INNOVACIÓN PÚBLICA HÍBRIDA. LABORATORIOS DE INNOVACIÓN}

La aparición de laboratorios de gobierno o de innovación pública es muy reciente, aunque ya existen varios ejemplos de referencia en todo el mundo. Estos laboratorios de innovación tienen varios rasgos en común, si bien se diferencian entre sí por el hecho de haber sido promovidos o no desde instancias gubernamentales. Por un lado, el origen se encuentra en un entorno universitario, como es el caso de GovLab de la Universidad de Nueva York. En otros casos, los laboratorios de innovación han sido directamente promovidos por determinados gobiernos, como es el caso del Laboratorio de Gobierno de Chile, de manera que tanto su financiación como el foco de su innovación se centra en la mejora del nivel de administración o gobierno correspondiente (aunque también puedan involucrar en sus dinámicas a otros niveles administrativos). Siguiendo esta primera lógica de diferenciación (origen universitario vs. origen gubernamental/administrativo), algunos de los laboratorios de innovación pública más reconocidos serían los siguientes.

- GovLab (Estados Unidos). Se trata un proyecto puesto en marcha por la Universidad de Nueva York. Está centrado en la aplicación de 
los avances tecnológicos al sector público con la filosofía del gobierno abierto y focalizado en los datos. Su método de trabajo consiste en el diseño de prototipos y su testeo controlado en entornos administrativos para adquirir aprendizaje que después puedan ser puestos a disposición de todo el sector público. Actualmente cuentan con numerosos proyectos abiertos en distintas administraciones públicas estadounidenses, destacando sobre todo aquellos relacionados con la aplicación práctica de los datos abiertos al sector público. GovLab cuenta con el respaldo de importantes compañías y asociaciones de la sociedad civil estadounidense e internacional, entre los que destacan la Fundación McArthur, la Fundación Knight y Google.

- MIT GOV/LAB (Estados Unidos). Es un laboratorio universitario dentro del Massachussets Institute of Technology, que tiene una orientación nítida hacía el desarrollo y contrastación de hipótesis sobre la rendición de cuentas y el compromiso, con el objeto de contribuir al conocimiento teórico y colaborar en el aprendizaje de los profesionales del sector público sobre cómo resolver los problemas públicos en tiempo real. En este caso, la conexión entre conocimiento teórico y aplicabilidad de soluciones se convierte en el eje de actuación.

- Gob Lab UAI (Chile). En este caso, se trata de uno de los primeros laboratorios de innovación pública en el ámbito universitario dentro del ámbito latinoamericano, creado en la Universidad Adolfo Ibáñez. Se trata de una experiencia muy reciente que pretende focalizarse en el conocimiento sobre el uso de Big Data en las políticas públicas chilenas. En concreto, se trata de un espacio donde desean plantear los grandes desafíos originados por la gran cantidad de datos e información que se generan por parte de las entidades gubernamentales.

- Laboratorio de Gobierno (Chile). El Laboratorio de Gobierno de la República de Chile, creado en 2014, es una iniciativa puesta en marcha por el Gobierno de Chile y se compone de un equipo multidisciplinar de varios ministerios. Se centra sobre todo en la ayuda en el diseño de servicios públicos y en la formación de sus empleados para reorientar las políticas a los intereses de los ciudadanos mediante la aplicación de métodos innovadores. Ha destacado en el ámbito de las políticas sanitarias a través de la iniciativa Impacta Salud (también liderada por el Gobierno de Chile). Además de con el respaldo del Gobierno chileno, el Laboratorio está formando alianzas con las instituciones de formación superior de su país mediante el programa AULAB.

- El LABgcba (Argentina). El Laboratorio de Gobierno de la Ciudad de Buenos Aires es una iniciativa del gobierno de la capital argentina dentro de su programa de gobierno abierto. Tiene varias líneas de 
trabajo, aunque en todas ellas existe una idea común: la localización de innovadores cívicos para canalizar sus proyectos a través del gobierno local y mejorar los servicios públicos de forma innovadora. Se trata de un laboratorio caracterizado por un fuerte liderazgo de cara a potenciar la idea de ecosistema de innovación y la generación de comunidad como plataforma para generar capacidades colectivas de acción.

- Laboratorio para la Ciudad (Ciudad de México). Este laboratorio de innovación pública también emerge desde la propia administración pública para facilitar la interacción entre la ciudadanía y las instituciones de gobierno. Se trata de una experiencia que destaca su propio carácter experimental, incluso, como espacio de especulación y ensayo, donde se pretende lanzar provocaciones para afrontar de manera diferente los problemas clave de la ciudad. También aquí se subraya la lógica de los proyectos piloto, así como el papel de la innovación social y la transformación urbana.

Junto con la categorización anterior, existen otros tipos de laboratorios que pueden orientarse a la solución de los problemas sociales. En el estudio de Acevedo y Dassen (2016) también incluyen como laboratorios de innovación pública a los denominados laboratorios de innovación social. En esta misma línea se sitúan los trabajos de Gascó (2017) o Tonurist, Kattel y Lember (2017), preguntándose estos últimos porqué los i-labs se crean en el sector público y testean hipótesis en la práctica. Así, los límites de los laboratorios de innovación pública son porosos, no solo en lo que respecta a su vinculación con el sector público, sino también en lo relacionado con la innovación social convencional. Al mismo tiempo, lo que todos parecen tener en común es el interés en promover un ambiente de innovación en las administraciones públicas o desarrollar innovaciones específicas en productos o servicios.

Todo ello pone de manifiesto que la innovación pública se encuentra en fase de desarrollo, también a través de laboratorios de innovación pública. En todos estos casos se pone de relieve la necesidad de incorporar el modelo de innovación abierta dentro del sector público. Los avances dentro de dinámicas de crowdsourcing mediante laboratorios de gobierno para la innovación pública se pueden plantear como una manera de caminar hacia modelos de servicio público que premian la creación colaborativa de soluciones a problemas públicos, transformando con ello las administraciones públicas en relación con su razón de ser (Seltzer y Mahmoudi 2012). A través de estas experiencias las organizaciones públicas descubren que la ciudadanía es una fuente nuclear de valor público y que 
cada vez que las personas afectadas contribuyen con una idea, sugerencia o iniciativa, las organizaciones públicas aprenden e incrementan su eficacia (Hilgers y Ihl 2010). Esa es la lógica a partir de la que se crea el caso que es el centro del presente artículo, tal y como se evidencia a continuación.

\section{ASPECTOS GENERALES DEL CASO. NOVAGOB.LAB EN EL ECOSISTEMA DE INNOVACIÓN PÚBLICA}

NovaGob.Lab pretende ser una síntesis de las mejores experiencias observadas en el ámbito internacional. Al mismo tiempo, el modelo de referencia que propone NovaGob.Lab se encuentra más cercano al GovLab o MIT GOV/LAB: pretende ser un espacio de innovación mediante la formulación de hipótesis y la utilización de teorías sobre problemas públicos, el diseño de prototipos para la generación de soluciones creando entornos de experimentación controlada, la capacitación a los empleados públicos para que creen valor y polo de atracción de innovadores públicos (una filosofía que dio inicio a NovaGob como red social) para aliarse mediante la creación de redes de colaboración con gobiernos y administraciones en el diseño y mejora de políticas públicas orientadas a la satisfacción de los intereses y derechos de los ciudadanos. A través del análisis de este caso, se pretende generar conocimiento sobre el papel y potencial de los laboratorios de innovación para mejorar la gestión pública.

El punto de Origen. NovaGob COMO ECOSistema de INNOVACIÓN PÚBLICA

Los ecosistemas de innovación sirven como terreno fértil para desarrollar innovación en las administraciones públicas. Para crear un ecosistema de innovación, son necesarias 4 condiciones (Figura 1) (Bason 2010): 1) enfocar consciente y sistemáticamente la renovación del sector público; 2) impulsar la capacidad innovadora en todos los niveles de gobierno; 3) liderar la innovación más allá del sector público; 4) incentivar procesos de co-creación. Cada una de ellas cuenta con un rol importante en la manera de entender la innovación pública como algo holístico y no solo centrado en determinadas personas, grupos o departamentos de una organización compleja. 
Figura 1: Factores necesarios para crear un ecosistema de innovación

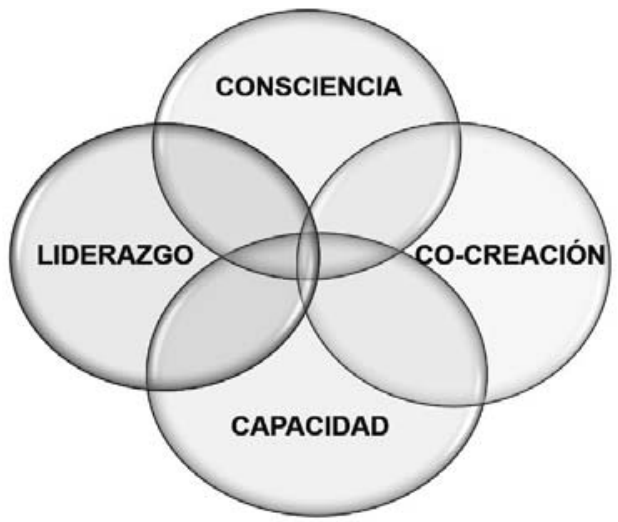

Fuente: Elaboración propia a partir de Bason (2010).

En primer lugar, la consciencia supone desarrollar el lenguaje de la innovación y trabajar para que los empleados públicos conozcan las implicaciones de la innovación y lo interioricen. En segundo lugar, la capacidad implica crear la estructura, dotar de recursos y organizar para innovar. Transformar la estructura organizativa burocrática en una innovadora. En tercer lugar, el liderazgo es fundamental para cualquier ámbito de la gestión pública, en el caso de la innovación el liderazgo debe basarse en la autoridad, la apertura y la colaboración. Superando la tradición jerárquica burocrática para generar liderazgos más horizontales e inclusivos. Finalmente, en cuarto lugar, la co-creación es un proceso activo, creativo y social: las denominadas 3 c de la co-creación, a saber 1) la conexión: interacción con los ciudadanos y actores, no solo provisión de servicios; 2) la colaboración: implicación activa de los ciudadanos en los procesos públicos, no solo consulta; y la 3) co-creatividad: creando nuevas soluciones con los ciudadanos y no para los ciudadanos. En conclusión, las administraciones públicas que quieran avanzar en la innovación deben crear espacios adecuados que faciliten, favorezcan y permitan la creatividad, la colaboración y la experimentación.

NovaGob en su conjunto es un proyecto para transformar la administración pública mediante la creación de una Comunidad Digital Iberoamericana de Innovación Pública. NovaGob surge como una spinoff de la Universidad Autónoma de Madrid (Espańa) en el año 2013 en el seno del Departamento de Ciencia Política y Relaciones Internacionales. Sus fundadores son profesores e investigadores que enfocan su trabajo a la mejora del uso de las tecnologías sociales en las administraciones públicas. Por lo tanto, se trata de un proyecto que tiene sus raíces en el ámbito 
universitario y enfocado a la transferencia de conocimiento científico.

La ciudadanía utiliza de manera masiva las redes sociales y las tecnologías móviles y además demanda contar con gobiernos más abiertos a la sociedad que usen de forma inteligente sus datos. La carencia de respuestas concretas a esta demanda está generando malestar social y baja valoración de las instituciones públicas. Por ello, cada vez más se propone el uso por parte de las administraciones públicas de tecnologías y plataformas sociales, que están demostrando su importante papel como herramientas de cambio social, para acercarse a la ciudadanía y mejorar su legitimidad social. Como expertos ofrecemos al sector público los servicios adecuados orientados para realizar el tránsito hacia ese cambio.

Uno de los principales objetivos de NovaGob consiste en la transferencia del conocimiento desde el mundo académico hacia la sociedad. Lo anterior se pretende lograr mediante la creación de soluciones concretas para su implementación en las administraciones públicas, y posteriormente recopilando el conocimiento empírico adquirido para su difusión en el ámbito académico. El proyecto cuenta con una lógica de crecimiento en red con varios nodos interconectados orientados a desarrollar sus correspondientes líneas de servicio.

La comunidad de innovación pública creada por NovaGob se compone por tres elementos fundamentales. Por un lado, novagob.org es la red social Iberoamericana de la Administración Pública. A mitad del año 2017, novagob.org contaba con más de 10.000 miembros provenientes de todos los países de la región iberoamericana. Une el talento para innovar y modernizar la administración a través de una plataforma 2.0. La red social es el germen del proyecto, que se ha convertido en el mayor nodo de encuentro, intercambio y colaboración de las empleadas y los empleados públicos del ámbito iberoamericano. En segundo lugar, el congresonovagob.com es el Congreso Iberoamericano de Innovación Pública. Es un encuentro anual que reúne a profesionales de la innovación pública, incluyendo talleres y diálogos sobre las mayores tendencias para la transformación de la administración pública. Durante este encuentro anual se entregan los Premios NovaGob Excelencia a aquellas administraciones públicas elegidas más innovadoras. El tercer nodo lo constituye NovaGob. Lab que es el Laboratorio de Gobierno para la Innovación Pública en Iberoamérica, cuya descripción se inicia en el siguiente apartado. 


\section{NovaGob.Lab, Laboratorio de Gobierno para la Innovación PÚBLICA EN IBEROAMÉRICA}

NovaGob.Lab es el primer laboratorio de innovación pública surgido en España. En concreto, y debido a su vinculación con la investigación universitaria en la Universidad Autónoma de Madrid (UAM), se caracteriza, sobre todo, por fomentar un ciclo de aprendizaje y contacto continuo entre el mundo académico y la gestión pública diaria. En primer lugar, empleando el conocimiento científico disponible para desarrollar hipótesis y generar prototipos viables, diseñar políticas y servicios públicos innovadores y posteriormente, tras su puesta en marcha, empleando la experiencia adquirida para verificarlos, completar o refutar hipótesis y teorías en el ámbito de la gestión pública. NovaGob.Lab pretende conseguir los siguientes objetivos:

- Constituir una red de instituciones y profesionales líderes en el ámbito de la innovación pública en Iberoamérica.

- Servir de puente entre el mundo académico y el sector público transfiriendo el conocimiento académico a los problemas prácticos y empleando el conocimiento empírico adquirido en la gestión pública para enriquecer la academia.

- Desarrollar y validar metodologías de innovación pública orientadas a la generación de valor público.

- Ayudar a las instituciones públicas a generar dinámicas de innovación continua para resolver nuevos problemas y generar valor público.

- Acelerar la innovación a partir de la creación de un hub, espacio de colaboración entre la academia, la ciudadanía y organizaciones públicas/privadas bajo un marco transparente y participativo.

- Crear comunidades de innovación localizando y formando a innovadores cívicos para emplear su conocimiento en la transformación de las instituciones públicas.

- Capacitar a los servidores y empleados públicos en materia de innovación pública.

La filosofía de trabajo de NovaGob.Lab se basa en el ciclo de innovación, permitiendo el desarrollo de distintas líneas de acción, de manera que puede entenderse como facilitador de las administraciones públicas para sus desarrollos más innovadores. El Laboratorio debe funcionar como un hub sobre innovación pública. Se trata de crear un espacio cívico de encuentro para el ecosistema de innovación pública que además sirve como sede física del laboratorio. Un lugar para el impulso de proyectos colaborativos para la innovación abierta, el intercambio de conocimiento, el networking, el mentoring, el desarrollo de jornadas y conferencias, 
y que además proporcionará espacios de coworking para académicos, profesionales del sector privado y empleados públicos. El vínculo del hub con la UAM ofrece una oportunidad para desarrollar su política de transferencia de conocimiento, así como un nodo de conexión con las organizaciones referentes en el ámbito de la innovación pública.

\section{LA POSICIÓN DEL LABORATORIO DE GOBIERNO EN LA TOMA DE DECISIONES. NOVAGOB.LAB EN ACCIÓN}

\section{La INNOVACIÓN PÚbLica EN ACCIÓN: EL CASO DE LA COMUNIDAD de Innovación en Planificación Estratégica para el Sector PÚBLICO}

El primer ejemplo del trabajo con dinámicas de laboratorio puesto en marcha por NovaGob.Lab se desarrolló junto con la empresa pública Ingeniería de Sistemas para la Defensa de España (Isdefe), adscrita al Ministerio de Defensa, de una Comunidad de Innovación en Planificación Estratégica para el Sector Público. El objetivo de la Comunidad era participar en el diseño de una herramienta de planificación estratégica para el sector público español, teniendo en cuenta una lógica de prototipado y la colaboración externa de actores relacionados con este ámbito de actividad dentro de las administraciones públicas.

El valor público que aportaron en este caso las dinámicas de laboratorio ha consistido por un lado en la creación de una comunidad de trabajo compuesta por diferentes perfiles. En concreto, la comunidad estaba formada por 14 profesionales del sector público (11 de diferentes unidades, agencias y empresas del sector público estatal con diferentes ramas de actividad y especialización y 3 de administraciones locales) a los que se sumaron otros expertos en planificación estratégica y otras materias como la comunicación, la tecnología o la participación provenientes de la sociedad civil y el mundo académico. Y por otra parte, la elaboración de prototipos y su testeo ha permitido localizar errores y espacios de mejora en la herramienta de planificación estratégica y reducir las posibilidades de aparición de fallos y errores de envergadura, algo que es especialmente delicado en el sector público. A continuación se detallan las fases de trabajo de la Comunidad (ver Tabla 1). 
Tabla 1: Esquema de trabajo de la Comunidad de Planificación Estratégica

Fase 1

Fase 2

Fase 3

\begin{tabular}{c|ccc|c}
\hline & & & & \\
$\begin{array}{c}\text { Creación de la } \\
\text { Comunidad }\end{array}$ & Sesión 1: & Sesión 2: & & \\
& Conceptualización & Diseño & Sesión 3: & $\begin{array}{c}\text { Testeo } \\
\text { amplio }\end{array}$ \\
& & y & Testeo inicial & y \\
& & Prototipado & & Difusión \\
\hline
\end{tabular}

Comunidad virtual

Fuente: Elaboración propia.

Fase 1: Creación de la Comunidad

Esta etapa consistió en el diseño de la Comunidad que iba a trabajar sobre el proyecto de planificación estratégica. Los perfiles de innovadores de la Comunidad debían cumplir con las siguientes características:

- Experiencia. Personas que en su trayectoria profesional hayan afrontado retos similares a los que se aborden en la sesión de trabajo.

- Especialización. Personas cuyos conocimientos fueran especialmente adecuados para abordar los retos identificados.

- Representatividad. Personas vinculadas a la comunidad de actores propia del reto a abordar.

El objetivo de esta fase consistía en que la comunidad de innovadores tuviese un perfil heterogéneo de modo que los grupos de discusión durante las sesiones de trabajo cuenten con diferentes perspectivas que ayudasen a elaborar diagnósticos, propuestas y prototipos innovadores que no se pueden generar en el entorno de trabajo habitual de los profesionales. En este sentido, se integraron en la Comunidad, además de los especialistas en planificación estratégica, que formaron el núcleo de la misma, perfiles del mundo de la comunicación pública, la participación, la tecnología y académicos especializados en Ciencia de la Administración. 


\section{Fase 2: Sesiones de trabajo de la Comunidad}

Con el fin de que la Comunidad pudiese intervenir en todas las fases de elaboración de la herramienta de planificación estratégica, se planificaron tres sesiones de trabajo con objetivos y metodologías distintas que se detallan a continuación.

La sesión 1 consistió en la conceptualización de las claves de gestión estratégica en las administraciones públicas. El objetivo fue reflexionar sobre los contenidos y funcionalidades básicas de una herramienta de planificación estratégica para el sector público. El método de trabajo seguido fue la creación de tres grupos de trabajo con temáticas diferentes relacionadas con la planificación estratégica: 1) análisis y diseño; 2) despliegue e implantación; y 3) evaluación. Los participantes debían realizar aportaciones argumentadas sobre dos asuntos: 1) elementos indispensables para realizar planificación estratégica en organizaciones públicas; y 2) funcionalidades básicas que debía proporcionar a sus usuarios una herramienta de planificación estratégica.

Las aportaciones de la Comunidad acabaron reflejadas en un mapa mental (Figura 2) que se empleó en futuras sesiones para diseñar portales específicos de la herramienta. Además, se diseñó un texto en formato wiki de modo que en el espacio virtual de la Comunidad sus miembros pudiesen ir realizando aportaciones con posterioridad a la sesión. 
Figura 2: Mapa mental de conceptos y funcionalidades básicas

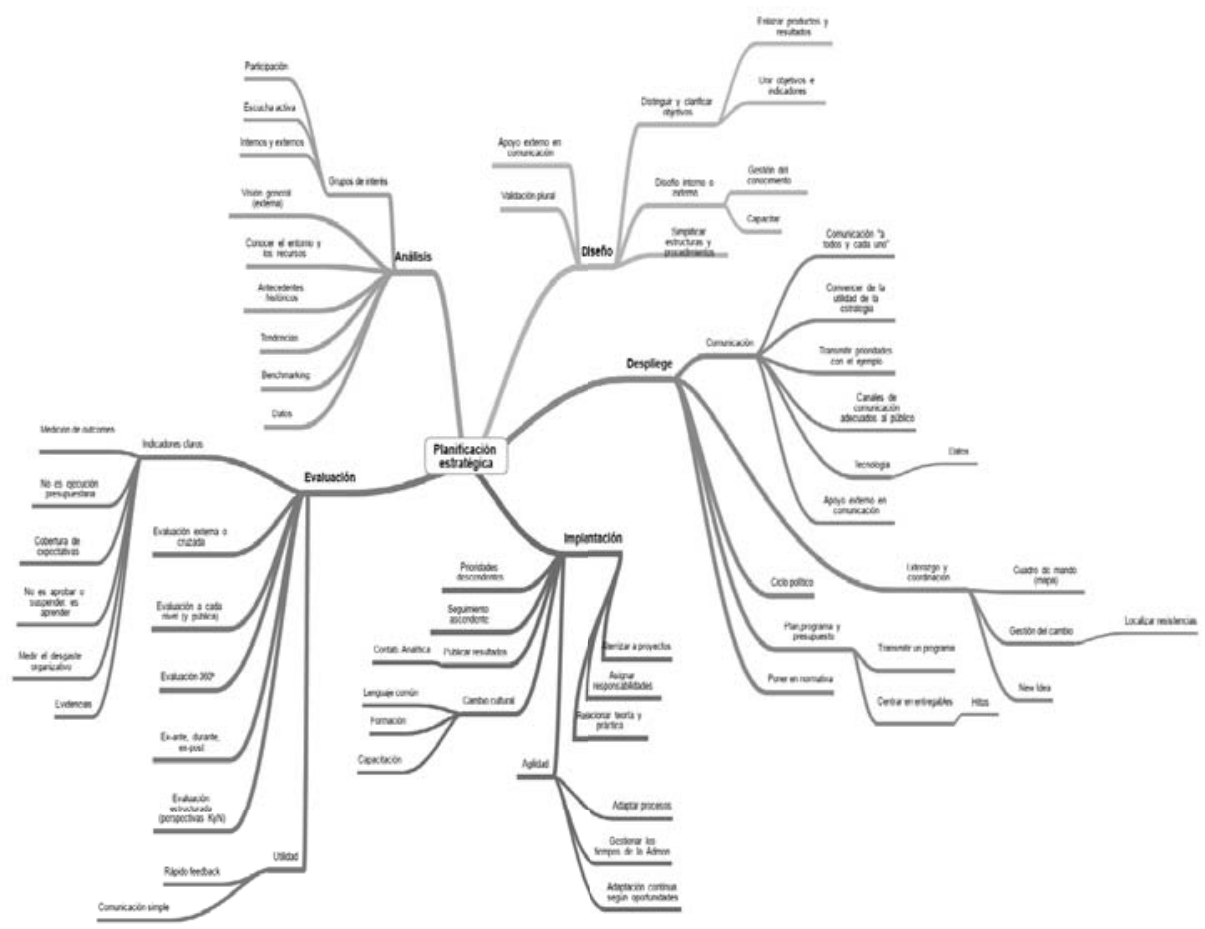

Fuente: Elaboración propia.

La sesión 2 se centró en el diseño y prototipado de portales de planificación estratégica. Su objetivo fue desarrollar los elementos críticos de la sesión anterior y diseńar y prototipar portales específicos de la herramienta de planificación estratégica. En términos de metodología se crearon tres grupos de trabajo para diseñar tres portales: 1) portal de comunicación del plan estratégico; 2) portal de participación del plan estratégico; y 3) portal del gestor. En una primera fase los grupos ponían en común las características y funcionalidades de cada portal para posteriormente, sobre el papel, diseñar las principales pantallas del portal sobre una lista de elementos previamente proporcionada por los organizadores. Una vez diseñado el portal, un equipo de técnicos informáticos realizó un prototipado de cada portal que fue mostrado a la Comunidad de modo que se pudiesen introducir cambios sobre esa primera versión.

El principal resultado fueron los prototipos semi-funcionales de cada portal diseñado por la Comunidad. Junto a estos prototipos se elaboró un documento en formato wiki con la descripción y los elementos de cada portal que los miembros de la Comunidad pudieron ampliar y matizar. 
La tercera sesión se dedicó al testeo de la herramienta de planificación estratégica. Se buscó testar los principales elementos de la herramienta de planificación estratégica, así como los portales diseñados en la sesión anterior con el fin de detectar posibles fallos y ámbitos de mejora.

Para ello, se crearon tres grupos de trabajo dedicados a los principales módulos de la herramienta de planificación estratégica: 1) análisis estratégico; 2) diseño estratégico; y 3) indicadores, cambios, proyectos y riesgos. Se planteó un ejercicio práctico sobre una organización pública a la que se adscribían los miembros de la Comunidad de modo que introdujesen información en la herramienta y testasen sus funcionalidades. Posteriormente se realizó una demostración a toda la Comunidad de las versiones finales de los portales diseñados en la sesión anterior.

El testeo permitió detectar un amplio número de posibles errores y espacios de mejora (más de veinte en la primera revisión) que se plasmaron en un documento que servirá de base para la elaboración de la fase beta de la herramienta. El documento contiene recomendaciones detalladas y gráficas de espacios de mejora realizados sobre la propia herramienta.

\section{Fase 3: Testeo amplio y difusión}

La última fase del trabajo de NovaGob.Lab respecto a la Comunidad de Innovación en Planificación Estratégica en el Sector Público tiene como objetivo la difusión de esta versión beta de la herramienta resultante de los trabajos anteriormente descritos entre administraciones y profesionales del sector público de modo que durante un periodo de tiempo más prolongado puedan probarla y realizar una relación de espacios de mejora para tener en cuenta a la hora de lanzar la versión definitiva. Esto permitirá un testeo más amplio que el realizado en las sesiones de trabajo y permitirá incorporar nuevas ideas y perspectivas. 
Figura 3: Muestra de la versión beta de la herramienta de planificación estratégica testada por la Comunidad

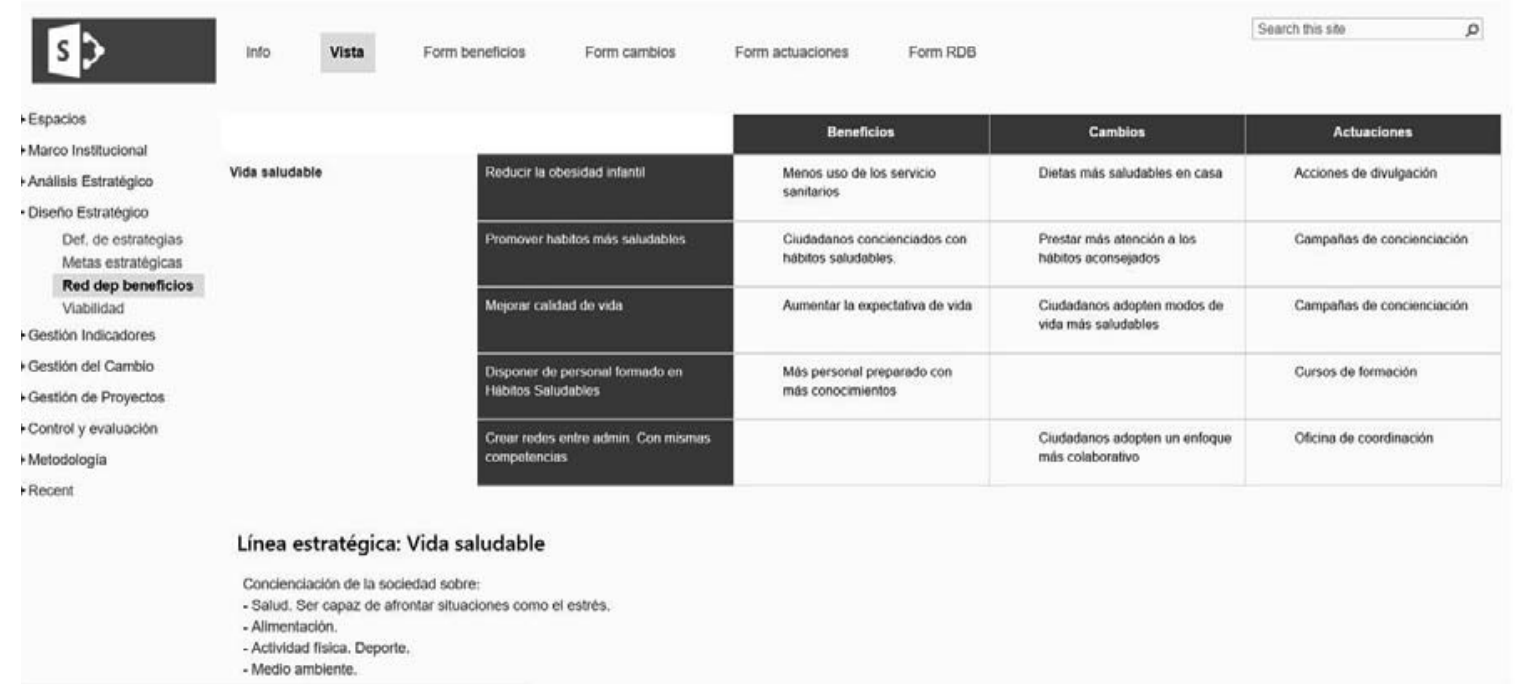

Fuente: Elaboración propia.

\section{CONCLUSIONES}

Este artículo ha puesto de manifiesto la importancia de la innovación pública, teniendo en cuenta el rol de los laboratorios de innovación. Inicialmente, se han sentado de las bases del cambio de paradigma que se encuentra detrás de la noción de innovación, superando enfoques de la NGP, en base a la puesta en práctica de la colaboración, la co-creación y la creación de valor público a través de integración de las personas en el proceso de creación de las decisiones y acciones públicas. Particularmente, se ha presentado el caso de NovaGob.Lab, laboratorio de gobierno para la innovación pública en Iberoamérica, el primero en su especie en el ámbito espańol. La presentación de este caso se ha realizado destacando sus oportunidades dentro de un contexto internacional en el que se han desarrollado recientemente experiencias cercanas en cuanto a objetivos, dinámicas, metodologías y resultados. El punto de interés entonces se refiere a la relación de este proyecto con NovaGob, como ecosistema de innovación pública, así como la existencia de diferentes proyectos asociados, sobre todo, la red social novagob.org o su congreso de innovación pública. En todo caso, este proyecto se encuentra muy estrechamente ligado a los innovation labs o living labs, tal y como se han identificado en la literatura reciente (Tonurist, Kattel y Lember 2017). En todos los casos, se pretende lograr innovaciones orientadas a solucionar problemas sociales concretos, a través de la utilización de datos masivos y la aplicación de metodologías de trabajo experimentales, abiertas al entorno de las organizaciones públicas. 
En relación con el caso de estudio, uno de los principales retos de las instituciones académicas es traducir su labor investigadora en iniciativas que supongan una mejora real en las comunidades a las que sirven. En este sentido, el caso NovaGob.Lab, mediante su método de trabajo basado en nuevas dinámicas de experimentación dentro de la gestión pública, supone un ejemplo claro de cómo la academia es capaz de proponer y desarrollar iniciativas que implican y afectan a toda la sociedad. En este sentido, resulta clave la existencia de un grupo de investigación sobre innovación pública y tecnologías en el gobierno y la gestión que promueve este proyecto. La investigación básica sobre innovación pública, gobernanza inteligente, gobierno abierto, redes sociales digitales, etc., genera hipótesis y propone teorías que se convierten en el punto de inicio de las propuestas de trabajo que después se contrastan en entornos reales. En otras palabras, se emplea la labor académica para servir a la sociedad y se usa la realidad concreta de la gestión pública para mejorar el conocimiento académico. Todo ello en un proceso de doble dirección que desea convertirse en referente a nivel nacional e internacional.

Otra de las conclusiones de este trabajo es que el crecimiento de los laboratorios de innovación pública es una oportunidad que se abre para gobiernos y administraciones públicas de todo el mundo para la experimentación ante la creciente complejidad de los problemas públicos. En unos casos, los laboratorios pueden ser promovidos por ellas mismas como espacios internos de desarrollo de ideas y dinámicas de colaboración. En otros casos, como espacios híbridos en los que interactuar con otras organizaciones con problemáticas comunes, así como situar a la ciudadanía en el centro. No obstante, estamos en un momento todavía incipiente en la implementación de la idea de innovación en el sector público (así como de los laboratorios), especialmente, en lo que respecta a su capacidad para alterar las dinámicas de poder asentadas en su seno desde hace décadas (de Vries, Bekkers, y Tummers 2016; Cejudo, Dussauge y Michel 2016). En efecto, lo anterior solo se podrá llevar a cabo a través de la aplicación de dinámicas de inteligencia colaborativa o crowdsourcing, así como la co-creación y co-producción de servicios y políticas públicas, generando procesos de gobernanza inteligente (smart governance) (Criado, 2016a; Noveck 2015).

En todo caso, el valor público creado por los laboratorios de innovación derivará de su capacidad para alterar las reglas de funcionamiento actuales, tanto formales como informales, así como los equilibrios de poder, no solo dentro de las organizaciones públicas, sino también en su relación con la sociedad. En efecto, el potencial de estos espacios de innovación pública parece todavía limitado en la medida que las experiencias en marcha han 
tenido un foco muy concreto, tanto en términos temáticos o áreas de políticas, como de alcance de las transformaciones propuestas, que se han centrado demasiado en procesos y menos en una dimensión conceptual o cultural del sector público. Como se ha apuntado antes, los laboratorios de innovación pública extenderán su capacidad de cambio en la medida que se conciban dentro de ecosistemas de innovación más amplios que involucren más actores y problemáticas sociales más complejas. 


\section{REFERENCIAS}

Acevedo, S. y Dassen, N. (2016). Innovando para una Mejor Gestión: La Contribución de los Laboratorios de Innovación Pública. Sin ciudad: BID.

Bason, C. (2010). Leading Public Sector Innovation: Co-Creating For A Better Society. Bristol: Polity Press.

Brugué, Q., Blanco, I. y Boada, J. (2014). Entornos y Motores para la Innovación en las Políticas Públicas. Revista del CLAD. Reforma y Democracia, 59, 5-34.

Brabham, D. C. (2013). Using Crowdsourcing in Government. Sin ciudad: IBM Center for the Business of Government.

Cejudo, G., Dussauge, M. y Michel, Cynthia. (Eds). (2016). La Innovación en el Sector Público: Tendencias Internacionales y Experiencias Mexicanas. Ciudad de México: INAP-CIDE.

Chesbrough, H. (2012). Open Innovation: Where We've Been and Where We're Going. Research-Technology Management, 55 (4), 20-27.

Clark, B. J., Brudney, J. y Jang, S. (2013). Coproduction of Government Services and the New Information Technology: Investigating the Distributional Biases. Public Administration Review, 73 (5), 687-701.

Criado, J. I. (2016a). Las Administraciones Públicas en la Era del Gobierno Abierto. Gobernanza Inteligente para un Cambio de Paradigma en la Gestión Pública. Revista de Estudios Políticos, 173, 245-275.

(Ed.). (2016b). Nuevas Tendencias en la Gestión Pública. Madrid: Instituto Nacional de Administración Pública.

Criado, J. I. y Villodre, J. (2016). Crowdsourcing y Plataformas Colaborativas en las Administraciones Públicas. En Criado, J. I. (Ed.), Nuevas Tendencias en la Gestión Pública. Madrid: Instituto Nacional de Administración Pública.

De Vries, H., Bekkers, V. y Tummers, L. (2016). Innovation in the public Sector: A Systematic Review and Future Research Agenda. Public Administration, 94 (1), 146-166. 
Gascó, M. (2017). Living Labs : Implementing Open Innovation in the Public Sector. Government Information Quarterly, 34 (1), 90-98.

Gil-Garcia, J. R., Zhang, J. y Puron-Cid, G. (2016). Conceptualizing Smartness in Government: An Integrative and Multi-Dimensional View. Government Information Quarterly, 33 (3), 524-534.

Hilgers, D. e Ihl, C. (2010). Citizensourcing: Applying the Concept of Open Innovation to the Public Sector. The International Journal of Public Participation, 4 (1), 67-88.

Linders, D. (2012). From E-Government to We-Government: Defining a Typology for Citizen Coproduction in the Age of Social Media. Government Information Quarterly, 29, 446-454.

Mergel, I. (2015). Open Collaboration in the public Sector: The Case of Social Coding on GitHub. Government Information Quarterly, 32 (4), 464-472.

Mergel, I. y Desouza, K. (2013). Implementing Open Innovation in the Public Sector: The Case of Challenge.gov. Public Administration Review, 73 (6), 882-890.

Noveck, B. S. (2015). Smart Citizens, Smarter State. Cambridge: Hardvard University Press.

Rivera León, L., Simmonds, P. y Roman, L. (2012). Trends and Challenges in Public Sector Innovation in Europe. Thematic Report 2012 under Specific Contract for the Integration of INNO Policy TrendChart with ERAWATCH (2011-2012) Contract number x07. Siu ciudad: Technopolis Group.

Rodríguez-Bolívar, M. P. R. y Meijer, A. J. (2016). Smart Governance: Using a Literature Review and Empirical Analysis to Build a Research Model. Social Science Computer Review, 34 (6), 673-692.

Seltzer, E. y Mahmoudi, D. (2012). Citizen Participation, Open Innovation, and Crowdsourcing: Challenges and Opportunities for Planning. Journal of Planning Literature, 28 (1), 3-18.

Sørensen, E. y Torfing, J. (2012). "Collaborative Innovation in the Public Sector. The Innovation Journal. The Public Sector Innovation Journal, 17 (1), 1-14. 
Tõnurist, P., Kattel, R. y Lember, V. (2017). Innovation Labs in the Public Sector: What They Are and What They Do? Public Management Review, 19 (10), 1455-1479.

Windrum, P. (2007). Innovation and Entrepeneurship in Public Services. En Windrum, P. y Koch, P. (Eds.), Innovation in Public Sector Services. Entrepreneurship, Creativity and Management. Cheltenham: Edward Elgar.

Recibido: 25-11-2016

Aceptación de la versión final: 16-05-2017 